\title{
Audio waves and its loss of energy in puncture needles
}

https://doi.org/10.1515/cdbme-2019-0006

\begin{abstract}
The location of a puncture needle's tip and the resistance of tissue against puncture are crucial information for clinicians during a percutaneous procedure. The tip location and needle alignment can be observed by image guidance. Tactile information caused by tissue resistance to rupture, allow clinicians the perception of structural changes during puncture. Nevertheless, this sense is individual and subjective. To improve percutaneous procedures, the implementation of transducers to enhance or complement the senses offer objective feedback to the user. Known approaches are e.g. based on integrated force sensors. However, this is connected to higher device costs, sterilization and certification issues. A recent publication shows the implementation of an audio transducer clipped at the proximal end of the needle. This sensor is capable of acquiring emitted sounds of the distal tiptissue interaction that are transmitted over the needle structure. The interpretation of the measured audio signals is highly depended on the transmission over the needle, the tissue and, the penetration depth. To evaluate the influence of these parameters, this work implements a simplified experimental setup in a controlled environment with a minimum of noise and without micro tremors induced by clinician's hands. A steel rod simulating a needle is inserted into pork meat of different thickness. A controlled impact covering the needle's tip mimics tissue contact. The resulting signals are recorded and analyzed for better understanding of the system.
\end{abstract}

Keywords: Percutaneous procedure, audio transducer, needle sensor, parametrization, classification of tissue, characterization of tissue.

\footnotetext{
*Corresponding author: Ivan Maldonado, Institute of Medical Technology, INKA, Otto-von-Guericke-University, Magdeburg, Germany, e-mail: ivan.maldonado@ovgu.de

Alfredo Illanes, Marco Kalmar, Thomas Sühn, Axel Boese and Michael Friebe, Institute of Medical Technology, INKA, Otto-vonGuericke-University, Magdeburg, Germany.
}

\section{Introduction}

Percutaneous procedures have proven to be efficient and safe during the extraction of a sample tissue for a diagnosis or a treatment that requires the administration of medication [1]. The main factors around the success and clinician's acceptance are its minimal discomfort on the patients, low cost, and precision. Last-mentioned, thanks to the implementation of medical imaging techniques as ultrasound or magnetic resonance.

Initially, the success of a percutaneous procedure implementing an imaging technique was associated with the clinician's skills and experience. Nevertheless, because its subjectivity, different researches in robotics [1][2], sensors [3][4], navigation [6], tracking [7] and enhancement algorithms [8] have shown to be objective, useful for clinicians during a procedure and, most of them successfully implemented on real scenarios. In the particular case of sensors, the majority of the applications remain on research and do not reach an operation room because regulations that make the approval of sensors extremely difficult. In most of the cases, sensors are located on the tip or any part of the needle's shaft in contact with tissue.

A recent publication [9] demonstrated that an audio transducer attached to the proximal out-patient end of a puncture needle is able to acquire relevant information generated from the interaction between the tip and tissue in the distal part. Until now, the concept of an audio transducer attached to a needle can identify the transition between two different types of tissue. But it is unclear how the acquired signal is influenced by the interaction between the needle and soft tissue and the transmission of vibroacoustic waves.

This work identifies parameters that help to understand the transfer function of the needle when it is located in two different depths inside muscle pork tissue. The identification of these parameters can be the first step for the characterization and classification of tissue. 


\section{Materials and Methods}

\section{Design of experiment}

The implemented experiment reproduces a needle procedure when its tip is located in three different depths inside bio tissue. During puncture, the needle's tip cuts fibre layers of the tissue. Due to the shape of the tip, this interaction causes longitudinal and radial forces that induce micro-vibrations [10][11]. These vibrations travel along the rigid needle's shaft. An audio transducer at the proximal end is capable of acquiring these vibrations as sound signals. The transmission of the micro-vibrations is expected to change when the depth of the needle increases. To prove that, an experimental setup was implemented.

From a mechanical point of view, a needle in a doctor's hand can be simplified as a free oscillating cantilever (see Figure ). When the tip is in contact with tissue, this model changes to the simply supported beam. Increasing the penetration depth reduces the free oscillating length of this beam and, increases the fixation or damping force on the distal end.

When an impact is given to the structure, the system starts to oscillate. Depending on the impact this oscillation is defined by a maximum amplitude and a damping constant of the system. These parameters should influence the acquired audio signal.

\section{Experimental Setup}

A steel rod behaves as a simplified model of a medical puncture needle (see Figure ). It was fixed horizontally and held from one end. The steel rod had a diameter of $1.5 \mathrm{~mm}$ and
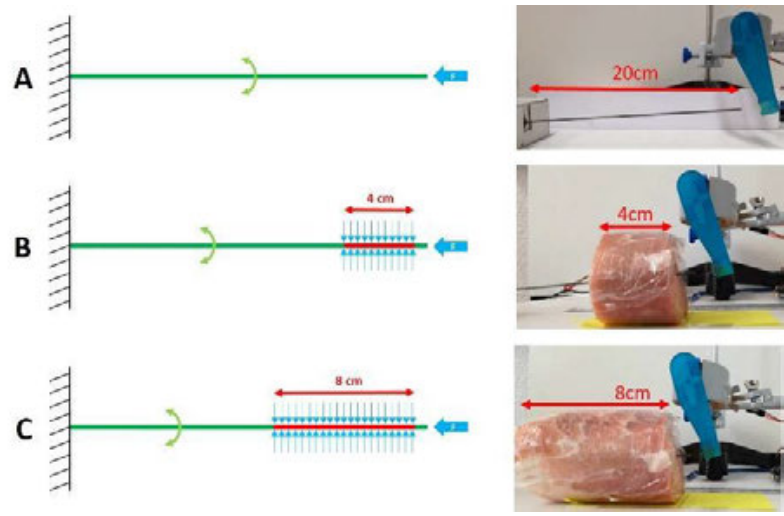

Figure 1: Simplified mechanical representation example. A) First scenario. Simple cantilever. B) Second scenario. Reduction of free oscillation in a length of 4 centimeters. C) Third scenario. Reduction of free oscillation in a length of 8 centimeters.

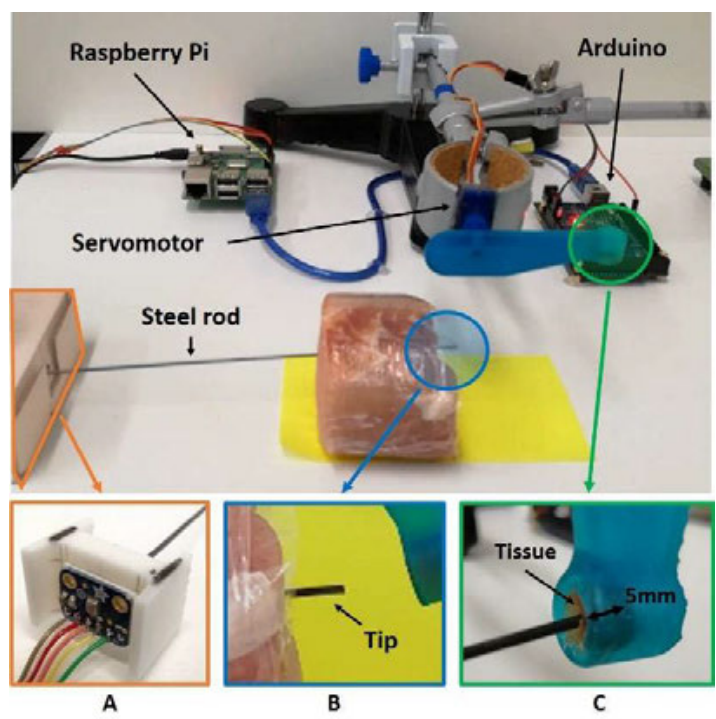

Figure 2: Experimental setup. A) Audio transducer attached to 3D holder with double tape. B) Steel rod's tip must be free for impact. C) Impact actuator. The maximum tissue pad is $5 \mathrm{~mm}$

a total length of $210 \mathrm{~mm} .10 \mathrm{~mm}$ of the proximal end of the rod were embedded to a 3D printed grip. An audio transducer (MEMS microphone SPH0645LM4H-B, Knowles, Illinois, USA) was fixed with double-sided tape with a centered hole to the 3D printed grip. The tape acts as environmental noise cancellation, in which the center hole allows sound waves to travel to the microphone. (see Figure A).

A Raspberry Pi 3 (Raspberry Pi Foundation, Cambridge, UK) computer stored and received all data acquired by the audio transducer via an $\mathrm{I} 2 \mathrm{~S}$ communication protocol. The implemented programming language to collect and store the data was written in Python 2.7.

An actuator was set to impact the needle's tip in a controlled manner. It consists of a servomotor (Analog servo 033212, JAMARA, Aichstetten, Germany) attached to a 3D printed hammer with a cavity at the tip. The cavity was filled with $5 \mathrm{~mm}$ of pork meat (see Figure C) for simulating the breaking through tissue layers during impact. An Arduino device (Arduino Mega 2560, arduino.cc, Somerville, U.S.A.) controlled the servomotor via Raspberry Pi commands.

For signal evaluation, a programming environment using MATLAB 2018b was created.

\section{Measurement Protocol}

Three different scenarios were considered. In the first scenario, the shaft of the rod was completely free; in the second and third scenario, the rod was inserted in 4 and $8 \mathrm{~cm}$ of pork meat respectively (see Figure and 2). The Python's software loaded at the Raspberry Pi device initialized the audio 

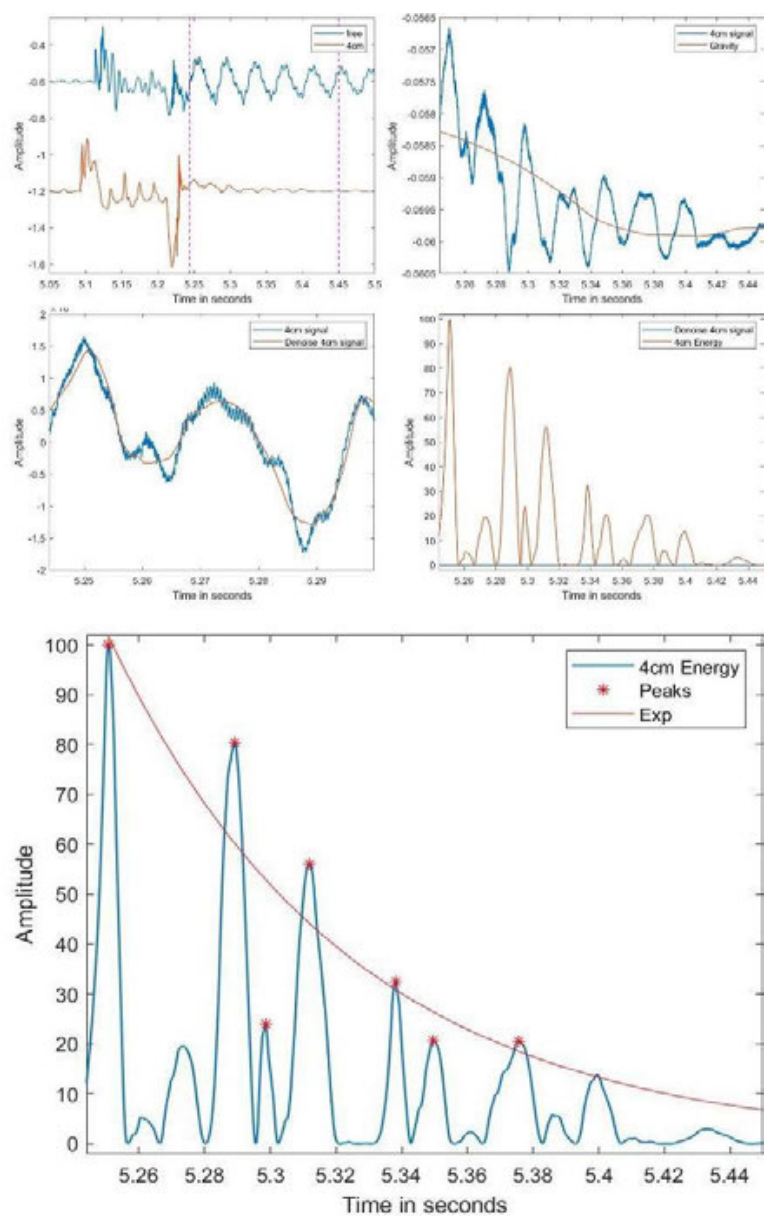

Figure 3: Signal processing. Left to right and up to down: a) manually selection; b) remove of gravity, c) denoising, d) instantaneous energy of signal and e) exponential fit procedure.

transducer recording and an internal timer. After 5 seconds, a command to a secondary logical program located at the Arduino initialized the actuator sequence that position the actuator twenty-five degrees from the tip, impact the needle's tip and return to its original position in a time of 0.2 seconds. It is important to clarify that the tip of the rod remains free in every moment to be able to receive the impact (see Figure B) and, the tissue set inside the $3 \mathrm{D}$ printer cavity had the same properties than the one surrounding the shaft.

Based on repeated experiments, it was possible to estimate that the actuator collided the needle's tip with a force of 1.5 N. All actuator's impacts were video recorded with a conventional Android smartphone at a resolution of FHD 1080p. The videos allow the visualization of the procedure for research and demonstration purposes.

The audio signals were stored as Waveform Audio File Format (WAV). Each audio file had a duration of 10 seconds with a sampling frequency of $44100 \mathrm{~Hz}$. In total, 60 audio signals, 20 for each scenario and each of them with a single measure, were acquired using an audio transducer.

\section{Signal Processing}

The main objective of the signal processing strategy was to compute significant parameters for characterizing the audio signal under different scenarios. For that, we assume that the signal resulting from the excitation impact can be parametrized using a simple damped sinusoidal model. For that, first, segments of 0.20 seconds were manually selected (see Figure 3a). This selection has as main objective to only take into account the signal segment corresponding to the needle response, avoiding influences of the impact per se or noise generated by the servomotor. Then, the influence of gravity was attenuated by trend removing using a multilevel one-dimensional discrete wavelet transformation with a Daubechies mother wavelet [12], (see Figure 3b). The resulting signal was then denoised using the same aforementioned wavelet transformation but with a higher frequency scale (see Figure 3c). The detrended and denoised signal was squared in order to estimate the instantaneous energy (see Figure 3d). Finally, the peaks of the energy signal higher than $10 \%$ of the maximum instantaneous energy were detected and used as data to be fitted by the exponential model (see Figure 3e)

$$
y^{\prime} \approx a \cdot e^{b \cdot x}
$$

where $a$ is the point of maximum instantaneous energy, $b$ is the constant of decay and, $y^{\prime}$ represents the peaks of maximum instantaneous energy consequence of the free oscillation induced in the system by simulating the breaking through of the tip.

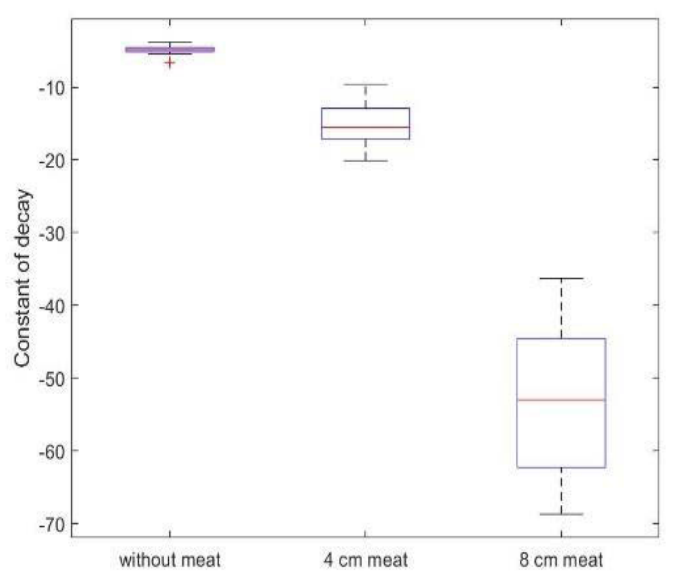

Figure 4: Boxplot from the parameter constant of decay. Each group is clearly differentiated from each other. 


\section{Results and Discussion}

The parameters of maximum instantaneous energy and constant of decay demonstrated to vary in correlation with the depth of the steel rod inside the pork meat (Table 1). The parameter of maximum instantaneous energy has a tendency to be more stable when the depth increase, opposite to the parameter constant of decay. The parameter of maximum instantaneous energy can be associated with waves of resonance meanwhile the constant of decay to the time of energy dissipation.

The parameter constant of decay shows a clear aggrupation of the different scenarios based on how quickly the energy dissipates (see Figure 4). It means that from the first point of maximum instantaneous energy, until the second peak of maximum instantaneous energy, the time varies in correlation with the tissue's depth. The values of this parameter do not overlap independently of their variance.

Table 1: Result of maximum instantaneous energy and constant of decay of 20 audio signals for each scenario.

\begin{tabular}{l|cc|cc}
\hline \multirow{2}{*}{ Scenario } & \multicolumn{2}{|c|}{$\begin{array}{c}\text { Maximum } \\
\text { Instantaneous Energy }\end{array}$} & \multicolumn{2}{c}{ Const. Of Decay } \\
& Average & Variance & Average & Variance \\
\hline Free & 93.90 & 42.50 & -4.87 & 0.38 \\
$4 \mathrm{~cm}$ meat & 70.73 & 135.57 & -15.05 & 12.32 \\
$8 \mathrm{~cm}$ meat & 97.03 & 5.70 & -53.53 & 100.30 \\
\hline
\end{tabular}

\section{Conclusions}

The audio signals follow the behavior of the mechanical model and, the parameters approached by an exponential form (Eq. 1) allow the differentiation of three scenarios in interaction with the same tissue.

The calculation of the parameters of maximum instantaneous energy and constant of decay contain information that indicates how fast the energy in the system dissipate based on the surrounding tissue. As future work, the system must be exposed to different tissues and their parameters must be analyzed. Advanced signal processing must be implemented to reduce ambient noise and micro tremors induced by the clinicians. The upcoming analysis must conclude if the information contained in the audio signals can be used for the extraction of useful features for a classification algorithm.

\section{Author Statement}

The Federal Ministry of Education and Research (BMBF) financially supported this research in the context of the INKA Project (Grant Number 03IPT7100X) and CONACYT Mexico. Authors state no conflict of interest. Informed consent has been obtained from all individuals included in this study.

\section{References}

[1] Vlastos, G., \& Verkooijen, H. M. (2007). Minimally invasive approaches for diagnosis and treatment of early-stage breast cancer. The oncologist, 12(1), 1-10.

[2] Kaya, M., Senel, E., Ahmad, A., \& Bebek, O. (2019). Visual needle tip tracking in 2D US guided robotic interventions. Mechatronics, 57, 129-139.

[3] Abayazid, M., Pacchierotti, C., Moreira, P., Alterovitz, R., Prattichizzo, D., \& Misra, S. (2016). Experimental evaluation of co-manipulated ultrasound-guided flexible needle steering. The International Journal of Medical Robotics and Computer Assisted Surgery, 12(2), 219-230.

[4] Tiwana, M. I., Redmond, S. J., \& Lovell, N. H. (2012). A review of tactile sensing technologies with applications in biomedical engineering. Sensors and Actuators A: physical, 179, 17-31.

[5] Puangmali, P., Althoefer, K., Seneviratne, L. D., Murphy, D., \& Dasgupta, P. (2008). State-of-the-art in force and tactile sensing for minimally invasive surgery. IEEE Sensors Journal, 8(4), 371-381.

[6] Zettinig, O., Frisch, B., Virga, S., Esposito, M., Rienmüller, A., Meyer, B., ... \& Navab, N. (2017). 3D ultrasound registration-based visual servoing for neurosurgical navigation. International journal of computer assisted radiology and surgery, 12(9), 1607-1619.

[7] Xia, W., Noimark, S., Ourselin, S., West, S. J., Finlay, M. C., David, A. L., \& Desjardins, A. E. (2017, September). Ultrasonic needle tracking with a fibre-optic ultrasound transmitter for guidance of minimally invasive fetal surgery. In International Conference on Medical Image Computing and Computer-Assisted Intervention (pp. 637-645). Springer, Cham.

[8] Mwikirize, C., Nosher, J. L., \& Hacihaliloglu, I. (2018). Signal attenuation maps for needle enhancement and localization in 2D ultrasound. International journal of computer assisted radiology and surgery, 13(3), 363-374.

[9] Illanes, A., Boese, A., Maldonado, I., Pashazadeh, A., Schaufler, A., Navab, N., \& Friebe, M. (2018). Novel clinical device tracking and tissue event characterization using proximally placed audio signal acquisition and processing. Scientific reports, 8(1), 12070.

[10] Barnett, A. C., Lee, Y. S., \& Moore, J. Z. (2016). Fracture mechanics model of needle cutting tissue. Journal of Manufacturing Science and Engineering, 138(1), 011005.

[11] Abolhassani, N., Patel, R., \& Moallem, M. (2007). Needle insertion into soft tissue: A survey. Medical engineering \& physics, 29(4), 413-431

[12] Bogusz, J., Klos, A., \& Kosek, W. (2013). Wavelet decomposition in the Earth's gravity field investigation. Acta Geodynamica et Geomaterialia, 10(1), 169. 